\title{
Adherence: the journey of medication taking, are we there yet?
}

\author{
Parisa Aslani $\cdot$ Marie Paule Schneider
}

Received: 27 November 2013/ Accepted: 27 November 2013/Published online: 6 December 2013

(C) Koninklijke Nederlandse Maatschappij ter bevordering der Pharmacie 2013

\begin{abstract}
Patient adherence to medications has been an issue challenging healthcare professionals for decades. Adherence rates, causes of non-adherence, barriers and enablers to medication taking, interventions to promote adherence, and the impact of non-adherence on health outcomes, have been extensively studied. In light of this, the area of adherence research has progressed conceptually and practically. This special issue contains a range of articles which focus on different aspects of adherence, from standardising terminology and methods of measurement, to non-adherence in a broad range of patient populations, and to interventions to promote adherence.
\end{abstract}

\section{Keywords Adherence $\cdot$ Intervention $\cdot$ Persistence}

Adherence to therapy amongst patients using chronic medications remains a challenge for patients and practitioners, despite the vast literature investigating the causes

P. Aslani $(\bowtie)$

Faculty of Pharmacy, The University of Sydney, Pharmacy and Bank Building A15, Broadway, NSW 2006, Australia

e-mail: parisa.aslani@sydney.edu.au

\section{P. Schneider}

Community Pharmacy, School of Pharmaceutical Sciences, University of Geneva, Geneva, Switzerland

\section{P. Schneider}

Community Pharmacy, School of Pharmaceutical Sciences, University of Lausanne, Lausanne, Switzerland

\section{P. Schneider}

Community Pharmacy, Department of Ambulatory Care \& Community Medicine, University of Lausanne, Lausanne, Switzerland of non-adherence, and numerous studies developing and implementing interventions to promote adherence. One of the key issues in medication taking is the dynamic nature of non-adherence, and the fact that it can be influenced by numerous factors, which can change over the course of a patient's medication taking journey. Compounding this complexity is the lack of a single intervention or even a combination of multiple strategies that can address the causes of non-adherence for a range of patients within a pot of an equally diverse mix of disease conditions. In parallel to tackling non-adherence on an individual patient basis, researchers and clinicians are also confronted with limitations in the tools available to reliably and validly assess patients' adherence levels as a first step in addressing nonadherence to therapy.

This special issue of the International Journal of Clinical Pharmacy bravely advances into the minefield of adherence and presents a range of articles focusing on different aspects of adherence, from standardising terminology and methods of measurement, to non-adherence in a broad range of patient populations, and interventions to promote adherence. The articles contribute to the evidence that the journey of adherence is variable for both individual and groups of patients, and presents challenges for healthcare professionals and researchers in identifying non-adherence, providing effective and tailored strategies, as well as monitoring and promoting adherence to therapy. Four key points underscore the articles included in this special issue:

- Adherence to therapy is a key factor in ensuring optimal clinical outcomes. All healthcare professionals, and specifically pharmacists, have an important role to play in identifying non-adherence, understanding patients' perspectives, delivering strategies to promote adherence, and monitoring patients' adherence to 
therapy. Delivering an interprofessional and collaborative adherence support service by all healthcare professionals involved in the care of a patient, can have a greater positive impact on improving patients' medication taking behaviour than if services are delivered by only one healthcare professional.

- There are many causes of non-adherence to therapy. These causes differ between patients as well as for individual patients over the life of their chronic condition. Although the evidence supporting the causes of nonadherence is strong, few studies have been able to satisfactorily address all potential causes of non-adherence. It is therefore not possible to determine and address individual patients' risk factors for non-adherence.

- Multifaceted interventions appear to be more effective at promoting adherence to therapy. However, interventions that address the key determinants of non-adherence for individuals, and which are therefore tailored to the individual's needs are more likely to be successful in improving adherence in the short and long term.

- There is a shift towards triangulation of adherence data collected by researchers, to increase the validity of adherence measurement methods. However, there is variation in the definitions of adherence in the literature, influenced by the instruments used and the conditions examined.

One of the limitations in the adherence literature is a lack of consistency in the terminology, and the use of a number of terms interchangeably. Moreover, cut-off levels which authors use to categorise adherence versus non-adherence, is inconsistent across the literature, preventing comparison of results between adherence studies. Ahmed and Aslani [1] have provided an overview of adherence-related terms used in the literature and their definitions. They highlight the importance of consistency in terminology and clear definitions of adherent behaviour, and together with other authors, advocate the use of internationally recognised, standardised definitions in research to allow for meaningful comparisons between studies conducted in this field [1,2].

The lack of uniformity in adherence definitions is further challenged by the range of tools available to assess adherence to therapy, due to a lack of a "gold standard" measurement tool. Lehman et al. [2] present a review of indirect measures of adherence which are routinely used to measure adherence to therapy in clinical practice and research studies. They observed that self-report measures of adherence are typically used to measure adherence at one point in time, whilst electronic tools provide an additional longitudinal measure of adherence, and are therefore useful for measuring implementation of, and persistence with therapy. Increasingly, prescription claims data are being used to determine the medication possession ratio and proportion of days covered for large cohorts of patients, and to triangulate self-report data. Whilst direct measures of adherence provide a more accurate and reliable measure of patients' medicine taking than indirect measures, Lehman et al. [2] recommend that triangulation of methods or the use of two or more indirect measures of adherence can increase the validity and reliability of adherence data collected in this way.

A few articles have focused on the factors impacting adherence [3-6]. For example, factors including patients' misconceptions about the disease condition and progression, experiencing and managing side effects, and opinions about the disease, were shown to influence adherence to imatinib in Taiwanese outpatients with chronic myeloid leukaemia [3]. Similarly, beliefs about medications had an impact on adherence to chronic medications used by pregnant women [7]. Lupattelli et al. [7] identified that during pregnancy, overall there was a low level of adherence to chronic medications, especially if the women had ceased treatment during a previous pregnancy without a negative impact on their health and if they believed that the medication was harmful to the foetus. Surprisingly, Gunther et al. [4] demonstrated that a patient's ethnicity did not influence their beliefs about treatment or their perceptions of illness, and therefore a patient's culture did not impact their adherence to therapy. These findings, however, contradict other research (e.g. [8,9]), and may be specific to the study sample and should be interpreted accordingly.

Fernandes et al. [5] evaluated adherence to short term antibiotics amongst patients in community practice. Whilst it is believed that adherence rates to short term therapy are higher than those witnessed during chronic therapy, Fernandes et al. [5] demonstrated that non-adherence to short term antibiotic therapy is high, and is influenced by factors such as difficulty in purchasing the antibiotic and swallowing the medication. In an interesting study examining the correlation between the use of over the counter (OTC) medications and adherence to prescribed medications in the elderly, Olesen and colleagues [6] highlighted that users of OTC medications were more likely to be adherent to prescription medications than the non-users.

It is well recognised in the literature that adherence to therapy is vital in improving clinical outcomes. Chen et al. [10] have reinforced these and demonstrated the positive impact of long-term adherence to imatinib on clinical treatment response and mortality in patients with chronic myeloid leukaemia [10]. Moreover, Gengiah et al. [11] have supported the importance of adherence to pre-exposure prophylaxis in a review of HIV prevention trials.

The literature is rich in evidence regarding the factors impacting adherence to therapy, as well as interventions which address these factors to promote both adherence and persistence. Whilst there is a shift towards multifaceted interventions 
in the literature, it is important to note that changes to treatment regimens such as reducing polypharmacy, decreasing the complexity of regimens, and reducing the cost implications for the patient [12] can have significant impacts on improving adherence. Moreover, one of the methods that can have the largest impact on adherence in elderly, especially in the ones who are less autonomous in managing their treatment, or the ones with complex regimens who forget to take their medications, or in instances of polypharmacy, is the use of multicompartment adherence aids [13].

However, there is still a gap in the availability of effective interventions that can have a sustained impact on adherence, and outside research settings. In an interesting commentary, La Caze et al. [14] discuss how to translate research, specifically regarding interventions to promote adherence, into clinical practice delivered by healthcare professionals to patients whom they suspect or know have poor adherence to their therapy. They advocate "targeted" and "tailored" interventions, which in essence means that healthcare professionals should have the skills and knowledge to deliver interventions targeted at patients they have identified to be non-adherent. Further, these interventions should be tailored for specific patients, addressing their barriers to adherence and their needs in order to be able to foster an improvement in their adherence to treatment and ensure they take their medications appropriately.

In summary, adherence research has progressed significantly. Conceptually, there is a greater push for internationally accepted standardised terminology and definitions. In research, there is a move towards triangulation of methods of measuring adherence, a call for robust methodology and development of multifaceted interventions to promote adherence to therapy. However, we are not at the end of the journey. There is a need for implementation of systems within primary healthcare to routinely assess and monitor patient adherence to therapy, identify non-adherence and provide targeted and tailored strategies to promote adherence. These systems should be capable of delivering such interventions which are embedded within a collaborative interprofessional patientcentred model of healthcare. Most importantly, the interventions developed should be evidence based. Ultimately, patients are responsible for their own medication taking, and it is reasonable to assume that every patient may be non-adherent at some point during their treatment. It is the responsibility of healthcare professionals to collaborate with each other and with the patient to identify factors that impact medication taking and implement agreed strategies to promote adherence.

Furthermore, it is time to translate effective interventions developed and evaluated in research studies into implementation strategies in routine care. Such implementation strategies should be targeted at several levels: the patient; the education of healthcare professionals; healthcare organizations, such as community and hospital pharmacy environments (management, human resources and settings), and the healthcare system level (reimbursement policies).

This special issue did not aim to include research in all aspects of adherence to therapy, and some emerging areas that were not covered are the impact of shared decision making, use of e-Health, multiprofessional collaborative interventions, and long term interventions on adherence; as well as key issues such as the cost-effectiveness analysis of interventions to promote adherence. New avenues and directions in adherence will always appear, and old areas are continuously revisited with new perspectives.

It therefore appears that the journey of adherence continues, and we are not quite there yet!

Funding None.

Conflicts of interest None.

\section{References}

1. Ahmed R, Aslani P. What is patient adherence? A terminology overview. Int J Clin Pharm. 2013. doi:10.1007/s11096-013-9856-y.

2. Lehmann A, et al. Assessing medication adherence: options to consider. Int J Clin Pharm. 2013. doi:10.1007/s11096-013-9865-x.

3. Chen L.-C, et al. Disease acceptance and adherence to imatinib in Taiwanese chronic myeloid leukemia outpatients. Int J Clin Pharm. 2013. doi:10.1007/s11096-013-9867-8.

4. Gunther M, et al. Treatment beliefs, illness perceptions, and nonadherence to antiretroviral therapy in an ethically diverse patient population. Int J Clin Pharm. 2013. doi:10.1007/s11096-013-9866-9.

5. Fernandes $M$, et al. Non-adherence to antibiotic therapy in patients visiting community pharmacies. Int J Clin Pharm. 2013. doi:10.1007/s11096-013-9850-4.

6. Olesen C, et al. Correlation between the use of 'over-the-counter' medicines and adherence in elderly patients on multiple medications. Int J Clin Pharm. 2013. doi:10.1007/s11096-013-9863-z.

7. Lupattelli A, Spigset O, Nordeng H. Adherence to medication for chronic disorders during pregnancy: results from a multinational study. Int J Clin Pharm. 2013 .doi:10.1007/s11096-013-9864-y.

8. Apter A, et al. Modifiable barriers to adherence to inhaled steroids among adults with asthma: it's not just black and white. J Allergy Clin Immunol. 2003;111:1219-26.

9. Staehelin C, et al. Longer term clinical and virological outcome of sub-Saharan African participants on antiretroviral treatment in the Swiss HIV Cohort Study. J Acquir Immune Defic Syndr. 2012;59(1):79-85.

10. Chen $\mathrm{T}-\mathrm{C}$, et al. Imatinib adherence associated clinical outcomes of chronic myeloid leukemia treatment in Taiwan. Int J Clin Pharm. 2013. doi:10.1007/s11096-013-9876-7.

11. Gengiah $\mathrm{T}$, et al. Adherence challenges with drugs for preexposure prophylaxsis to prevent HIV infection. Int J Clin Pharm. 2013. doi:10.1007/s11096-013-9861-1.

12. Reeve E, Wiese M. Benefits of deprescribing on patients' adherence to medications. Int J Clin Pharm. 2013. doi:10.1007/ s11096-013-9871-z.

13. Mosca C, et al. Assessing the impact of multi-compartment compliance aids on clinical outcomes in the elderly: a pilot study. Int J Clin Pharm. 2013. doi:10.1007/s11096-013-9852-2.

14. La Caze A, Gujral G, Cottrell WN. How do we better translate adherence research into improvements in patient care? Int J Clin Pharm. 2013. doi:10.1007/s11096-013-9869-6. 